\title{
Sociología
}

Sordini, M. V. (2019). Una lectura sociológica sobre los programas alimentarios en América del Sur. Revista de Sociología, 34(2), 1-19. doi: 10.5354/0719529X.2019.54255

\section{Una lectura sociológica sobre los programas alimentarios en América del Sur}

\author{
[A sociological assessment of food programs in South America]
}

\author{
María Victoria Sordini (i)
}

CONICET - Universidad Nacional de Mar del Plata

\begin{abstract}
Resumen
El objetivo de este trabajo es analizar los programas alimentarios implementados en diez países de América del Sur en 2017-2018 en relación con la situación alimentaria de la región. El diseño del estudio es cualitativo de tipo exploratorio. Desde el enfoque de la etnografía virtual se analizaron sesenta y seis programas alimentarios de la Plataforma para la Seguridad Alimentaria y Nutricional. Se profundiza la observación en los objetivos, población objetivo y prestación. Los objetivos de las políticas públicas brindan pistas para pensar cuál es el problema del hambre y cuál es la sociedad deseable hacia la que estas intervenciones se orientan; la lectura sobre las modalidades de prestación permite reconocer las maneras de responder a la problemática y observar la población objetivo en las que se focaliza la política. Entre los resultados obtenidos se destaca que el problema alimentario se cristaliza en el aumento de la obesidad en general, y en particular en las mujeres de bajos ingresos, sin embargo, un bajo porcentaje de los programas trabaja sobre esa cuestión.
\end{abstract}

Palabras clave: programas alimentarios, malnutrición, políticas sociales, América del Sur.

\begin{abstract}
The present work constitutes an exploratory qualitative review of the food policies implemented in ten South American countries between 2017 and 2018, taking into consideration the nutritional situation of the region. The virtual ethnography approach was used to analyze sixty-six food policies from the Food and Nutrition Security website. Observation focused on the goals, target population and provisions of each policy. An assessment of the goals raises questions as to the nature of the problem of hunger and exactly what type of society such programs seek to mold; a reading of the modes of provision reveals the strategies used to address the problem, and an observation of the target population points to the sectors of society upon which the policies are focused. One of the most important findings is that the food crisis is manifested in a general increase in obesity, particularly among women on lower incomes. However, only a small percentage of the programs address this issue.
\end{abstract}

Keywords: food programs, malnutrition, social policies, South America.

Contacto: La comunicación sobre este artículo debe ser enviada a María Victoria Sordini, email: mvsordini@hotmail.com.ar 


\section{INTRODUCCIÓN}

Cada plato de comida cristaliza las relaciones sociales de producción, de distribución, de comercialización y las prácticas de consumo que permiten la constitución del mismo y atraviesan al comensal. Las acciones y decisiones que emanan estas relaciones sociales intervienen directamente sobre las condiciones de vida, de reproducción de la vida y de la fuerza de trabajo de todos los sectores sociales. En el contexto mundial, desde los años 70 la disponibilidad de alimentos ha ido en aumento. Sin embargo, esta excedencia no garantiza el acceso y las condiciones de desigualdad sostienen que amplios sectores sociales de diversos países sigan soportando la escasez de alimentos y la insuficiencia de nutrientes. En términos estadísticos, en 1985 se alcanzó la disponibilidad plena de alimentos para todos los habitantes del mundo, sin embargo, ese año la Organización de las Naciones Unidas para la Alimentación y la Agricultura (conocida como FAO) registro 875 millones de desnutridos; en la actualidad, con una disponibilidad excedentaria, los desnutridos alcanzan a los 1,000 millones (Aguirre, 2015).

En esta tensión aparece la intervención estatal sobre la cuestión social, mediante las políticas sociales, modulando dichas desigualdades. La política social en general, y las políticas alimentarias, en particular están ligadas de forma recursiva a la estructura social, en tanto surge de esta y configura los procesos de estructuración social (Adelantado, Noguera, \& Rambla, 2000). Este trabajo propone observar los programas alimentarios implementados en diez países de América del Sur (Argentina, Bolivia, Brasil, Chile, Colombia, Ecuador, Paraguay, Perú, Uruguay y Venezuela) y ponerlos en tensión con la situación alimentaria de la región. A partir de los datos que ofrecen la páginas web de la Plataforma para la Seguridad Alimentaria y Nutricional (SAN, 2019a), creada por la Comunidad de Estados Latinoamericanos y Caribeños (CELAC), se construyó una matriz cualitativa que detalla los componentes de sesenta y seis programas alimentarios registrados: objetivo, población objetivo, cantidad de destinatarios, modalidades de prestación, inicio temporal del programa, alcance de la cobertura geográfica, organismo responsable, fuente de financiamiento y criterios de egreso/salida del programa. En este trabajo se profundiza el análisis sobre los objetivos, los destinatarios y las modalidades de intervención que plantean los programas.

Desde una mirada sociológica sobre las políticas sociales (Adelantado et al., 2000; Boito \& Huergo, 2011; Cena, 2014; De Sena, 2011; Garretón, 1999; Grassi, 2003; Scribano \& De Sena, 2013) se analizarán los objetivos, población objetivo y modalidades de prestación de los programas en relación a la cuestión alimentaria en la región (Aguirre, 2005; FAO, 2014; FAO \& Organización Panamericana de la Salud [OPS], 2017). De esta manera, se observarán las continuidades y rupturas entre el problema alimentario regional y las modalidades de intervención de las políticas alimentarias.

¿Qué es una política alimentaria? 

los programas alimentarios en América del Sur. Revista de Sociología, 34(2), 1-19. doi: 10.5354/0719529X.2019.54255

La igualdad ciudadana nunca es completa, está reñida por la desigualdad económica, constituyendo un horizonte que no alcanza a materializarse. En esta tensión aparece la intervención estatal ligada de forma recursiva a la estructura social, en tanto surge de esta y configura los procesos de estructuración social, constituyendo un bucle de influencia recíproca (Adelantado et al., 2000). Así, el diseño, contenido y modalidades de implementación de las políticas sociales actúa de modo recursivo con la dinámica del ámbito mercantil, doméstico y relacional. Las intervenciones asignan cómo redistribuir recursos en la sociedad desde cada esfera y moldear las relaciones sociales que se establecen entre ellas permitiendo las condiciones para cambios, permanencias estructurales o la emergencia de nuevos actores sociales (Adelantado et al., 2000).

Las políticas sociales hacen sociedad en tanto producen las condiciones para la existencia de la sociedad como tal. Según Garretón (1991) tales condiciones se alcanzan en determinados niveles de igualdad socioeconómica, con una calidad de vida acorde a la diversidad cultural y, la existencia de actores sociales que negocien con autonomía la representación de la ciudadanía en las esferas políticas y estatales. Desde la mirada de Danani (2009), las políticas sociales hacen sociedad porque a través de sus intervenciones se expresan y construyen los modos de vida y las condiciones de reproducción de la vida. Una sociedad define sus sujetos, objetos y medios para satisfacer las necesidades en un proceso sociopolítico, institucional, económico y cultural en el que se construye la política social.
Las políticas alimentarias, al intervenir sobre las necesidades y demandas sociales que constituyen a la cuestión social, consolidan los contornos de lo que el Estado delimita como problema alimentario (Grassi, 2003). Su arbitraje plasma las modalidades de responder a la cuestión instalando los límites de su acción y las condiciones de sus destinatarios. En las prestaciones alimentarias se materializa la capacidad de intervenir y transformar la realidad que subyace en los enunciados de sus diseños.

La actuación estatal pone a disposición determinados nutrientes visibilizando $u$ ocluyendo las deficiencias en la alimentación de la población. Los límites energéticos corporales que garantizan las intervenciones estatales configuran los límites de las energías disponibles, de la producción y reproducción de algunos tipos de fuerza de trabajo, de determinados desarrollos cognitivos, interacciones sociales y trayectoria de clase (Sordini, 2017). Por ello las políticas alimentarias, en tanto políticas de los cuerpos, remiten a las formas y condiciones socialmente aceptadas para disponer de las personas, manteniéndolas en los límites energéticos para sobrevivir (Scribano \& De Sena, 2013).

Los requisitos para acceder a las prestaciones pautan las categorías de inclusión o exclusión en los destinatarios en relación con sus prácticas alimentarias y condiciones de vida. La acción del Estado define los requisitos que determinan a una persona como receptora y merecedora de un programa alimentario (Grassi, 2003). La focalización de las políticas sociales se 

los programas alimentarios en América del Sur. Revista de Sociología, 34(2), 1-19. doi: 10.5354/0719529X.2019.54255

implementa en la región desde los años 80 con el argumento de optimizar la eficiencia para estimular la equidad bajo el financiamiento de los organismos multilaterales de crédito (Sojo, 1990). Sin embargo, la focalización atenta contra las metas de equidad al atender a una población puntual bajo el riesgo de generar una selectividad imperfecta y fragmentar los lazos sociales diferenciando entre quienes acceden y quienes no acceden a los programas sociales (De Sena, 2011).

La cuestión alimentaria en la región de América del Sur

La transición nutricional se caracteriza por cambios cualitativos como cuantitativos de la dieta. Los cambios alimentarios adversos incluyen una dieta con mayor densidad energética, lo que significa más grasa y más azúcar añadido en los alimentos, una mayor ingesta de grasas saturadas (principalmente de origen animal) unida a una disminución de la ingesta de carbohidratos complejos y de fibra, y una reducción del consumo de frutas y verduras. Estos cambios alimentarios se combinan con cambios del modo de vida que reflejan una reducción de la actividad física en el trabajo y durante el tiempo de ocio (Organización Mundial de la Salud [OMS] \& FAO, 2003).

Mientras los sectores sociales con mejores ingresos modifican su transición nutricional hacia el aumento de proteínas de origen animal (Aguirre, 2015; OMS \& FAO, 2003), los sectores sociales de menores ingresos moldean sus dietas en torno a las posibilidades de acceso (Aguirre,
2005). En este escenario el mercado produce alimentos baratos para la distribución masiva, pero con exceso en grasas saturadas, azúcar y sodio (Aguirre, 2011) profundizando así el consumo de alimentos ricos en carbohidratos y en grasas, carentes en fibras y proteínas (Aguirre, 2005). La vida urbana condiciona el acceso a los alimentos según el poder adquisitivo de las personas en el mercado. Tanto los precios de los alimentos como los ingresos disponibles son determinados por las leyes del mercado y regulados por la intervención estatal (Aguirre, 2005; Grassi, Hintze, \& Neufeld, 1994).

En ambos extremos sociales aparecen las condiciones para una ingesta excesiva de grasas, tanto por un consumo excesivo de proteínas de origen animal, o por un consumo excesivo de alimentos baratos que brindan más saciedad y menos nutrición. Este contexto predispone a la prevalencia de sobrepeso, obesidad y hambre oculta. La obesidad interviene como factor de riesgo de enfermedades crónicas no trasmisibles asociadas a la nutrición, algunas de las cuales son causas importantes de muerte en la región, por ejemplo, la enfermedad isquémica del corazón, la diabetes mellitus no insulinodependiente o de Tipo II, la hipertensión arterial, algunos tipos de cáncer, la osteoartritis y la osteoporosis, entre otras (Peña \& Bacallao, 2000).

Las carencias de nutrientes se ocluyen en los cuerpos fornidos que consumen productos alimenticios ultra-procesados con baja calidad nutritiva. En otras palabras, se come, pero no se nutre. El hambre oculta (De Castro, 1955) es determinado por las comidas monótonas o el 
consumo de alimentos concentrados o purificados en los cuales predominan determinados componentes químicos y resultan escasos otros micronutrientes. Las causas de sobrepeso y obesidad en la región se relacionan a la ingesta de productos ultra-procesados con altos contenidos de azúcar, grasa y sal, a la disminución de las preparaciones culinarias tradicionales con alimentos frescos, el estilo de vida sedentario, los procesos de urbanización sin un planeamiento para una movilidad más activa de las personas, la jornada laboral extensa, la desregulación del mercado y la publicidad de productos alimenticios no saludables (FAO \& OPS, 2017).

El consumo de alimentos con baja calidad nutritiva ha aumentado rápidamente en los países de ingresos medios bajos desde 1980, al observar la obesidad en relación a la situación económica, en las poblaciones adultas se desplaza a los sectores sociales con menores ingresos y con mayor prevalencia en las mujeres (Monteiro, Moura, Conde, \& Popkin, 2004; OMS, 2011). Siguiendo los datos de FAO (2017), en América Latina y el Caribe 24 países presentan una proporción de personas obesas en valores cercanos o superiores al $20 \%$ de la población. En América del Sur, las cifras más altas se encuentran en Argentina dónde cerca del 29\% de la población es obesa y en Chile se alcanza al $30 \%$. En cambio, países como Bolivia y Paraguay presentan tasas inferiores al $20 \%$ de la población.

Al observar el crecimiento en las tasas de obesidad en los países de América del Sur entre 1980 y 2014 para hombres y mujeres, según datos de OMS (FAO \& OPS, 2017), en las mujeres el aumento es más intenso. Mientras en 1980 todos los países se mantienen por debajo del $10 \%$ de la población de varones, con la excepción de Argentina (12.5\%), Uruguay (10.5\%) y Chile (10\%), en 2014 todos los países alcanzan y/o superan el $15 \%$ de la población de varones, excepto por Ecuador (14.5\%), Bolivia (12.4\%) y Guyana (13.9\%). Esto indica un aumento en la tasa de obesidad en varones en los países de América del Sur. En cambio, al observar las tasas de obesidad en mujeres en 1980 los países de la región superan el 15\% con la excepción de Paraguay (6.5\%), Bolivia (8.5\%) Ecuador (9.1\%) y Brasil (9.5\%) y, en 2014 todos los países superan el $20 \%$ de su población femenina con obesidad. Sin embargo, se destacan Argentina, Guyana, Uruguay y Chile con más del 30\% de mujeres con obesidad. Esta cifra no alcanza en ningún país la población masculina marcando una fuerte prevalencia de esta patología en las mujeres. (FAO, 2017).

Mientras las mujeres registran mayor prevalencia al sobrepeso y obesidad que los hombres, un $33 \%$ de las mujeres en edad fértil padece anemia en todo el mundo (FAO, Fondo Internacional de Desarrollo Agrícola [FIDA], OMS, Programa Mundial de Alimentos [PMA] \& Fondo de las Naciones Unidas para la Infancia [UNICEF], 2017). La malnutrición femenina y su capacidad gestante constituyen en el útero materno el inicio de trayectorias de vida hacia la malnutrición (Aguirre, 2005). Esta condición durante la gestación y los primeros años de vida desarrollará infancias afectadas por la desnutrición o el sobrepeso que afectarán el 
desarrollo cognitivo, óseo y dental de los niños/as. En la población infantil mientras el sobrepeso y la obesidad afecta a 41 millones de niños menores de cinco años en el mundo, la desnutrición crónica (i.e., retardo de altura para la edad), asociada normalmente a situaciones de pobreza, y relacionada con dificultades de aprendizaje y menos desempeño económico (FAO, 2014), impacta en 155 millones de niños menores de cinco años en 2016. Es importante mencionar que entre 2005-2016 este último trastorno descendió de $29.5 \%$ a $22.9 \%$. También prevalece la desnutrición aguda (i.e., deficiencia de peso por altura) asociada con periodos recientes de hambruna o enfermedad que se desarrolla muy rápidamente (FAO, 2014). Así, se estima que la desnutrición aguda afecta a 52 millones de niños menores de cinco años en 2016 (FAO et. al., 2017).

La imposibilidad para adquirir alimentos suficientes durante al menos un año se define como subalimentación, ello implica un nivel de ingesta por debajo de las necesidades de energía requerida. En 2016 el número de personas subalimentadas en el mundo aumentó hasta los 815 millones en comparación con los 777 millones de 2015, sin embargo, las cifras siguen siendo inferiores a los 900 millones de personas subalimentadas registradas en el año 2000 (FAO et. al., 2017). En la Tabla 1 se esboza la prevalencia de subalimentación en el periodo 2000-2016 visualizando las tasas a nivel mundial, en América Latina y el Caribe y en América del Sur.

Tabla 1: Prevalencia de la subalimentación 2000-2016 a nivel mundial, en América Latina y Caribe y en América del Sur en el periodo 2000-2016 (\%)

\begin{tabular}{lccccccccc}
\hline & 2000 & $\mathbf{2 0 0 5}$ & $\mathbf{2 0 1 0}$ & $\mathbf{2 0 1 1}$ & $\mathbf{2 0 1 2}$ & $\mathbf{2 0 1 3}$ & $\mathbf{2 0 1 4}$ & $\mathbf{2 0 1 5}$ & $\mathbf{2 0 1 6}$ \\
\hline Mundo & 14.7 & 14.2 & 11.5 & 11.2 & 11 & 10.8 & 10.7 & 10.6 & 11 \\
América Latina y el Caribe & 12.0 & 9.1 & 6.8 & 6.6 & 6.4 & 6.3 & 6.3 & 6.3 & 6.6 \\
América del Sur & 11.1 & 8.0 & 5.9 & 5.7 & 5.5 & 5.4 & 5.4 & 5.5 & 5.9 \\
\hline
\end{tabular}

Nota: Elaboración propia según datos FAO (2017)

Todas las regiones presentan una tendencia al descenso de modo constante hasta 2015. En América del Sur la prevalencia de subalimentación descendió aproximadamente a la mitad, acompañando la tendencia mundial y de América Latina y el Caribe. Al desglosar las cifras de Mesoamérica, Caribe y América del Sur, esta última registra la menor prevalencia de subalimentación (FAO et al., 2017). En la Figura 1 se ilustran las cifras de subalimentación que algunos países de América del Sur registraron en el período 2014-2016 para observar la situación intrarregional. 


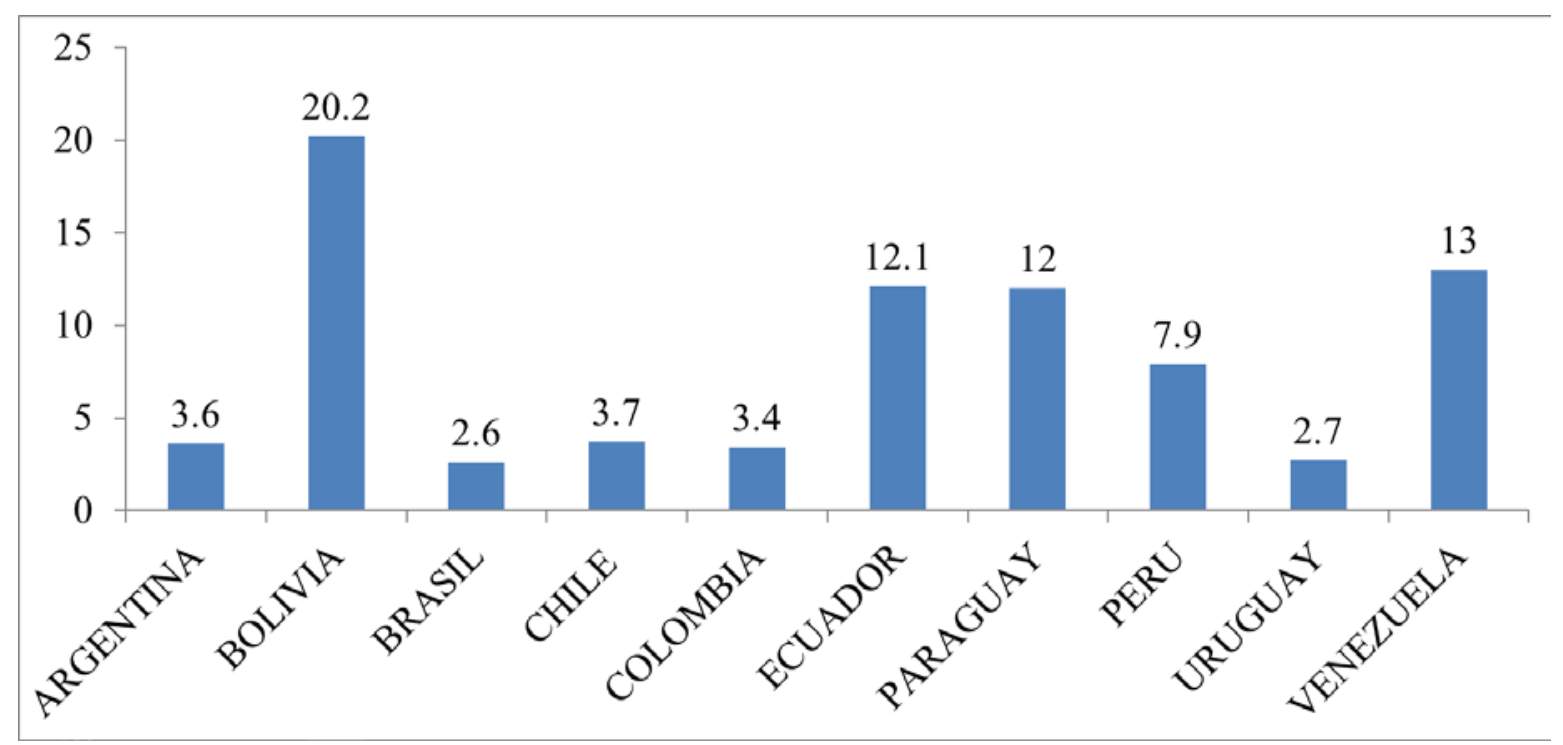

Figura 1. Prevalencia de la subalimentación (\%) en países de América del Sur en el periodo 20142016. Elaboración propia a partir de datos de FAOSTAT, 2017. Los datos disponibles en FAOSTAT sobre la prevalencia de subalimentación para Uruguay pertenecen al periodo 2008-2010 y para Brasil el periodo 2007-2009

Las tasas más altas las presenta Bolivia con un $20.2 \%$ de prevalencia de la subalimentación en la población en el periodo 2014-2016. Al observar la tasa de obesidad de 2014 para este mismo país, en hombres registra el menor porcentaje (12.4\%) $\mathrm{y}$ en mujeres registra el segundo menor porcentaje (23.1\%).

En el extremo opuesto, Argentina tiene la menor tasa de subalimentación en 2014-2016 con un $3.6 \%$ y representa la tasa más alta de obesidad en hombres $26.7 \%$ y en mujeres el $30.1 \%$ en mujeres. Este dato posiciona a Argentina entre los cuatro países con mayores tasas de obesidad en mujeres en la región en 2014, junto a Chile (32.8\%), Uruguay (31.3\%) y Guyana (30.3\%) (FAO, 2017). En cambio, los países con la más alta subalimentación de la región corresponden a Venezuela (13\%), Ecuador (12.1\%) y Paraguay
(12\%) continúan, luego de Bolivia. Sin embargo, Venezuela también se posiciona entre las más elevadas tasas de obesidad en hombres $(21.9 \%)$ y en mujeres (29.4\%).

En resumen, desde los años dos mil se puede observar una tendencia al descenso de subalimentación y un marcado incremento en las tasas de sobrepeso y obesidad, particularmente en mujeres. Por lo tanto, esta investigación observa las intervenciones de los programas alimentarios porque en sus diseños subyace el modo de definir a la cuestión alimentaria como cuestión social. Es decir, a partir de observa los objetivos, la población objetivo y las modalidades de prestación de los programas se ponen en tensión sus alcances, discontinuidades y rupturas con el panorama alimentario de la región. 


\section{MÉTODO}

Para alcanzar los objetivos propuestos, se generó una investigación empírica en base a un diseño cualitativo de tipo exploratorio. Para observar los programas alimentarios implementados en América del Sur se implementó el enfoque de etnografía virtual a través del acceso a fuentes de información en internet para el análisis de sus textos y contenidos (Hine, 2004). En este trabajo se utilizó, como fuente secundaria, la Plataforma para la Seguridad Alimentaria y Nutricional que es construida por CELAC, con el apoyo de la FAO y la Asociación Latinoamericana de Integración (ALADI) para disponer de un sistema de información sobre políticas públicas e indicadores que permite caracterizar los elementos que han contribuido a los avances de América Latina y el Caribe en la erradicación del hambre en 2017-2018.

La muestra de programas alimentarios es intencional a partir de los registros disponibles en la Plataforma para la Seguridad Alimentaria y Nutricional en los siguientes países de América del Sur: Argentina, Bolivia, Brasil, Chile, Colombia, Ecuador, Paraguay, Perú, Uruguay y Venezuela. Se sistematizó la información publicada sobre sesenta y seis programas alimentarios en una matriz cualitativa que discrimina cada uno de sus componentes, a saber: objetivo, población objetivo, cantidad de destinatarios, modalidades de prestación, inicio temporal del programa, alcance de la cobertura geográfica, organismo responsable, fuente de financiamiento y criterios de egreso/salida del programa.
En este trabajo se profundiza el análisis sobre tres dimensiones: (a) objetivo de la política; (b) población objetivo de la política; y (c) modalidad de prestación de la política. La selección de estas dimensiones se fundamenta a partir del recorrido teórico esbozado en el primer apartado de este trabajo. En primer lugar, los objetivos de las políticas públicas brindan pistas para pensar cuál es el problema del hambre y cuál es la sociedad deseable hacia la que estas intervenciones se orientan. En segundo lugar, realizar una lectura sobre las modalidades de prestación permite reconocer las maneras de responder a la problemática. Finalmente, observar la población a la que están dirigidos los programas permite advertir los sectores sociales en los que se focaliza la intervención y cuáles son los grados de alcance y cobertura de las intervenciones.

Los objetivos de las políticas públicas se operacionalizaron a partir de identificar las acciones principales de cada propuesta, de esta manera se construyeron las siguientes categorías: (a) mejorar el rendimiento escolar; (b) luchar-combatir-superar la pobreza; prevenir o tratar carencias nutricionales; (c) reducir sobrepeso y obesidad; (d) promover hábitos saludables; (e) mejorar condiciones de agricultura familiar; (f) apoyar el derecho a la alimentación; (g) fortalecer organizaciones de la sociedad civil; (h) promover desarrollo infantil integral; (i) contribuir al acceso de alimentos; (j) impulsar la industria alimenticia/ garantizar 
proveer políticas alimentarias para pequeños productores.

El proceso de operacionalización realizado para la categoría "mejorar el rendimiento escolar", por ejemplo, incluye a todos los programas que convergen en esta acción principal de su objetivo general, como por ejemplo el Programa de Alimentación Escolar (PAE) de Chile que plantea como objetivo general:

Mejorar la asistencia clases y retener a los estudiantes en el sistema educacional, a través de la entrega de un servicio de alimentación saludable, variado y nutritivo que permita un desarrollo físico $\mathrm{y}$ mental que favorezca $\mathrm{y}$ estimule la concentración y aprendizaje necesario para su éxito académico (SAN, 2019b). La dimensión población objetivo de la política se desagrego según la definición de requisitos para ser destinatario de cada intervención, pudiendo ser: (a) población en general; (b) hogares urbanos y rurales; (c) niños hasta 18 años (desagregados en menores de seis años y en edad escolar a nivel primario y secundario); (d) mujeres embarazadas y/o lactantes; (e) organizaciones de la sociedad civil; (f) pacientes en servicios públicos de salud/personas con discapacidad; (g) emprendedores y pymes; y/o (h) adultos mayores. La dimensión modalidades de prestación se operacionalizó al observar la principal prestación de cada intervención, a saber: (a) comedor escolar; (b) comedor; (c) complemento alimentario; (d) capacitación; (e) promoción y acceso al mercado de alimentos; (f) autoproducción; (g) financiamiento de proyectos/apoyo técnico; (h) transferencias monetarias; (i) promoción de la salud; (j) compras comunitarias; (k) bono para alimentos; y/o (l) disponibilidad y acceso a la actividad física/diagnóstico nutricional.

Si bien todos los países plantean diferencias en sus gestiones de gobierno, situación de la economía, conflicto social, modalidades de producción de alimentos, recursos naturales, superficie, población etc., la lectura transversal de las intervenciones alimentarias, lejos homogenizar criterios de análisis, pretende advertir algunos puntos de contacto para pensar el abordaje de la situación alimentaria regional. Se registraron seis programas de Chile, diez intervenciones en Brasil, ocho programas de Argentina, seis programas de Bolivia, tres intervenciones en Colombia, dos programas de Perú, ocho programas alimentarios de Ecuador, ocho intervenciones en Uruguay, nueve programas en Venezuela y, finalmente, seis programas de Paraguay. El nombre de cada programa se detalla en el anexo.

Sistematizar esta información permite realizar una lectura sociológica que tensione los objetivos propuestos por las políticas alimentarias vigentes, los modos de materializar sus intervenciones y la cuestión alimentaria de la región en la actualidad. 


\section{RESULTADOS}

Los objetivos: ¿Para qué son las políticas alimentarias?

Los objetivos de la política indican los contornos de la intervención, señala la definición del problema alimentario y el alcance de la solución propuesta. Siguiendo los registros mencionados las acciones predominantes en cada objetivo refieren a impulsar, mejorar, complementar, incentivar, promover, prevenir y fomentar en determinados sectores sociales su situación alimentaria hacia otros estadios. De este modo, la política social modera las condiciones de desigualdad pues:

Se refiere a las elecciones entre objetivos y fines políticos en conflicto y su formulación; analiza aquello que constituye una buena sociedad o aquella parte de la buena sociedad que distingue culturalmente entre las necesidades y aspiraciones del hombre social en contradicción con las del hombre económico (Titmuss, 1981) La acción que aparece con mayor frecuencia, representando un $28 \%$ del registro, es contribuir al acceso a los alimentos. Estas intervenciones se materializan con la modalidad de prestación predominante: complemento alimentario (caja/bolsón de alimentos secos) en tanto esta representa un $22 \%$ de las estrategias de la totalidad de los programas.

En segundo lugar, el objetivo con mayor presencia es la prevención o tratamiento de las carencias nutricionales que representa un $15 \%$ de las metas planteadas. La categoría incluye tanto a las carencias nutricionales ocasionadas por déficit (subalimentación o desnutrición) o por exceso en la ingesta (hambre oculta, obesidad), sin embargo, en los programas prima la intervención sobre las carencias por la falta. Por ejemplo, el Programa multisectorial desnutrición cero de Bolivia se propone "erradicar la desnutrición en niños y niñas menores de cinco años implementando intervenciones integrales, fortaleciendo la estructura $y$ funcionalidad multisectorial a niveles departamentales y municipales" (SAN, 2019c, "Objetivo general", parr. 1). Y el Programa de atención al riesgo nutricional en Uruguay busca "contribuir a mejorar el estado de salud y nutrición de menores de 18 años, mujeres embarazadas y en lactancia, que se encuentran en riesgo de malnutrición por déficit o desnutridos" (SAN, 2019d, "Objetivos", parr. 1). Estos objetivos orientan la acción de manera específica en la desnutrición y ninguno hace referencia a las carencias nutricionales que genera la malnutrición y ocluye la obesidad.

En el panorama nutricional de la región la subalimentación tiene menor incidencia que la obesidad. Sin embargo, la categoría reducir el sobrepeso y obesidad representa solo el 5\% de las intervenciones. En este caso, Bolivia también propone una intervención específica con el programa multisectorial de alimentación y nutrición en el ciclo de la vida con el que propone "contribuir a la reducción de la obesidad y sobrepeso en toda la población, contribuir a la reducción de la desnutrición en el ciclo de vida de las personas y, promover la implementación de 

los programas alimentarios en América del Sur. Revista de Sociología, 34(2), 1-19. doi: 10.5354/0719529X.2019.54255

estrategias de alimentación y nutrición" (SAN, 2019e, "Objetivos específicos", parr. 3-5).

Por otro lado, realizar una lectura sobre los objetivos permite advertir cómo se posiciona a los destinatarios en la escena del conflicto. $\mathrm{Al}$ respecto, un $12 \%$ de las intervenciones plantea el objetivo de promover hábitos saludables proyectando un diagnóstico de la cuestión alimentaria en la que predominan los hábitos no saludables en las personas. Aquí la política social mientras estigmatiza y controla, oculta las relaciones de la cuestión alimentaria con el contexto global (Faleiros, 2000). Si bien las poblaciones urbanas sostienen prácticas alimentarias que fomentan la malnutrición, el acto de comer es atravesado por cuestiones macroeconómicas (disponibilidad, autonomía, sustentabilidad de la producción), el acceso en relación a la capacidad adquisitiva dependiente del Estado y del mercado (Aguirre, 2005; Grassi et al., 1994), prácticas culturales (tradiciones y costumbres), estrategias domésticas de consumo como abastecimiento, y elaboración de alimentos (Aguirre, 2005), la construcción social del gusto que delimita los alimentos deseables de los accesibles y las representaciones de cuerpo saludable. Entones, al enunciar como meta promover hábitos saludables se diluyen las responsabilidades gubernamentales, de una problemática estructural y multi-causal, en los comportamientos individuales de los malnutridos, asignando las causas y soluciones de la cuestión alimentaria mediante procesos de individualización (Cena, 2014).

Entre los objetivos con mayor presencia continúan mejorar el rendimiento escolar y luchar-combatir-superar la pobreza, representando un $7 \%$ de las intervenciones cada uno. Los objetivos de mejorar las condiciones de la agricultura familiar y apoyar el derecho a la alimentación representan un $6 \%$ de las intervenciones cada uno. Los programas que proponen como acción principal impulsar la industria alimenticia, garantizar proveer políticas alimentarias para pequeños productores y promover la lactancia materna representan un $5 \%$ cada uno de las intervenciones en la región; los que proponen como acción principal promover el desarrollo infantil integral representan un $3 \% \mathrm{y}$, finalmente los programas que tienen por objetivo general fortalecer organizaciones de la sociedad civil representan un $1 \%$ de los programas alimentarios registrados. En esta lectura priman las intervenciones de corte compensatorio que complementan las dietas de la población.

Las prestaciones: ¿Cómo se logran las metas?

Leer los objetivos requiere de observar las estrategias mediante las cuales se materializan las intervenciones. El complemento alimentario (e.g., caja/bolsón de alimentos secos) es la modalidad más reiterada en los programas analizados. Para los presupuestos y los costos de logística es conveniente la intervención alimentaria con entregas de alimentos secos en detrimento de alimentos frescos (Aguirre, 1990). Desde los años 90 se toma un giro en la modalidad de intervención de las políticas sociales en América Latina mediante las transferencias de ingresos (Cecchini \& Atuesta, 

los programas alimentarios en América del Sur. Revista de Sociología, 34(2), 1-19. doi: 10.5354/0719529X.2019.54255

2017), años más tarde se extiende esta modalidad al campo de las políticas alimentarias. Actualmente las transferencias monetarias para el consumo de alimentos representan un $11 \%$ de las intervenciones de los programas registrados en este trabajo.

Algunos autores reconocen en la modalidad de transferencias monetarias cierta autonomía en la elección de alimentos dentro de las posibilidades de compra condicionada por los montos y los comercios/cadenas de supermercado habilitados con el sistema posnet (Britos et al., 2015). Es pertinente subrayar cómo la bancarización de la prestación direcciona las fechas en las que se realizan los consumos, los lugares habilitados para realizar las compras, los tipos de productos que componen las ofertas, tanto en cantidad de productos iguales, como en segundas marcas o calidad de los alimentos. De este modo se delimita el acceso a los alimentos en las leyes de la oferta y la demanda, se ubica a los alimentos como mercancía y a los destinatarios como consumidores (Sordini, 2018). En este este escenario solo un $2 \%$ de los programas analizados ofrecen prestaciones de compras comunitarias, denotando el predominio de intervenciones individualizadoras.

La segunda prestación que predomina en un $19 \%$ de los programas alimentarios registrados desempeña las dinámicas de capacitaciones y talleres. En el marco de actividades comunitarias se trasmiten estrategias vinculadas a las prácticas alimentarias que orientan la acción hacia hábitos saludables, sin carencias nutricionales, con soberanía alimentaria, con una dieta completada por los productos otorgados. Se trata de reuniones en las que los destinatarios participan de charlas y actividades sobre aptitudes para acercarse cada vez más a ser una persona saludable, según los parámetros de la intervención. A su vez, en un $10 \%$ de los programas su intervención se enfoca en la promoción de la salud.

Entre las modalidades de prestación menos frecuente en la región, un $2 \%$ garantiza la disponibilidad y acceso a la actividad física y a un diagnóstico nutricional individual. Esta intervención se vincula con la prevención y tratamiento de la obesidad, ya que la actividad física permite contraponer los efectos de los hábitos sedentarios de la vida urbana, tratar la ingesta excesiva de grasas de origen animal y ultra procesados. Esta intervención se aproxima a trabajar sobre uno de los problemas de malnutrición más importantes de la región.

Las prestaciones vinculadas a ofrecer comedor escolar representan un $10 \%$ de las intervenciones, comedor comunitario un $6 \%$, promoción y acceso al mercado de alimentos un $4 \%$, autoproducción de alimentos un $8 \%$, financiamiento de proyectos/ apoyo técnico representa un 6\% de las intervenciones.

La población objetivo: ¿para quién es la intervención?

Al observar a la población objetivo de cada programa se trata de intervenciones predominante focalizadas en sectores sociales en condiciones de pobreza. En este contexto, la política alimentaria se aleja de un rol integral y multisectorial para focalizar sus recursos en los 
sectores más empobrecidos: $30 \%$ hogares, desagregados en urbanos y rurales; un $36 \%$ para niños hasta 18 años, desagregados en menores de seis años y en edad escolar (nivel primario y secundario); $9 \%$ mujeres embarazadas y/o lactantes.

El $14 \%$ de los programas está dirigido a la población en general esto denota que sus objetivos se vinculan a una perspectiva trasversal a todos los sectores sociales. Sin embargo, ante un $69 \%$ de focalización en sectores de bajos ingresos se denota cómo el avance de la obesidad en todos los sectores sociales se solapa bajo un problema de poblaciones empobrecidas. Los restantes destinatarios representan: organizaciones de la sociedad civil $1 \%$, pacientes en servicios públicos de salud/personas con discapacidad $2 \%$, emprendedores y pymes $4 \%$, adultos mayores $4 \%$.

\section{DISCUSIÓN Y CONCLUSIONES}

El hambre es la marca de pertenencia a la intervención estatal, es el cuerpo del malnutrido el que cristaliza las contradicciones estructurales en las que se origina y se configura la política alimentaria. En el recorrido de este trabajo se buscó discutir las distancias entre la situación nutricional de la población de la región y los programas alimentarios que se implementan.

El objetivo más reiterado en la región (i.e., contribuir al acceso de alimentos) y la modalidad de prestación implementada con mayor frecuencia (i.e, entrega de caja/bolsón de alimentos), ofrece pistas para pensar las complejidades y contradicciones mediante las cuales los Estados definen el problema alimentario como una falta que es complementada/compensada. La reiteración de la fórmula problema/solución legitima la naturalización de hambre/caja o bolsón de alimentos secos instalando una imagen que normaliza y normatizan las intervenciones (Grassi, 2003).

Mientras amplios sectores sociales no comen lo que saben o lo que quieren, sino lo que pueden (Aguirre, 2005) las prestaciones de talleres y capacitación sobre la alimentación son menos primordiales que aquellas que garantizan el acceso. En la dinámica de taller en la que los destinatarios asisten para aprender y conocer estrategias vinculadas a la cuestión alimentaria la auto-responsabilidad se hace cuerpo. Lejos de diagramar un panorama multicausal e intersectorial de la cuestión alimentaria, numerosos programas refuerzan la intervención sobre los comportamientos individuales. Mediante talleres y capacitaciones se posiciona el lugar del hambre en el ámbito doméstico y se trasladan recursos para resolver la cuestión alimentaria mediante la familiarización/ comunitarización (e.g., mediante la agricultura familiar), privatizando la responsabilidad en la 
esfera familiar y alejando al Estado como garante del derecho (Danani, 2009).

Los contornos que delimitan la cuestión alimentaria a partir de la intervención de los programas la ubican como un problema de la pobreza siendo los Estados quienes modulan la desigualdad (Adelantado et al., 2000). La política alimentaria se involucra poco en intervenciones universales que garanticen a toda la población el derecho a la alimentación. Los recursos disponibles se dirigen a complementar la falta, la carencia y la ausencia. Los límites de intervención de cada programa alimentario sobre la población destinataria "pobre" define de modo oficial y legítimo tal condición. Los requisitos para el acceso al programa construyen una imagen de sí mismos en tanto receptor del programa y de los otros. La intervención alimentaria proyecta una imagen de la cuestión alimentaria, su impacto en la salud y las prácticas de alimentación que definen una pertenencia cultural. En este escenario, las energías que garanticen las prestaciones serán las que la estructura social dispone para el desempeño de la vida de la sociedad, por ello los programas alimentarios hacen sociedad.

Reflexionar sobre los modelos de sociedad deseable que subyacen en los diseños de los programas alimentarios implica poner en tensión el panorama alimentario de la región y sus intervenciones. A partir de los resultados se observa que mientras la obesidad ha incrementado desde 1980 con mayor incidencia en sectores de bajos recursos y en las mujeres, resultan escasas las intervenciones dirigidas a reducir el sobrepeso y la obesidad en la población en general, y en las mujeres en particular. Lo mismo sucede con las intervenciones dirigidas a embarazadas y lactantes, que son menores en comparación a otros destinatarios, al considerar que las trayectorias de los niños vinculados con la mala alimentación se inician en el útero materno.

La complejidad del entramado de acciones para el diseño, gestión e implementación de los programas delimita los grupos de población sobre los que se enfocan las estrategias, señala y condiciona qué y cómo comer, moldea formas de socializar en torno a la inscripción del programa y recepción de las prestaciones. En esta clave, se configuran formas de ser receptor de un programa alimentario, modos de actuar en relación a sí mismo y a los otros, se habilitan/deshabilitan determinados alimentos, se configuran modos de comensalidad y, se normatizan los modos de experimentar el hambre.

El trabajo se realizó a partir de una lectura sociológica desde los datos disponibles en la Plataforma para la Seguridad Alimentaria y Nutricional, construida por CELAC, FAO y ALADI. Este aspecto es tanto una limitación como una potencialidad. En primer lugar, el análisis se limita a los datos disponibles en estas fuentes de información, sin embargo, permiten realizar una lectura transversal sobre los diez países en relación a las políticas públicas que han intervenido sobre la problemática alimentaria en 2017-2018. Lejos de homogeneizar los contextos sociopolíticos, económicos y geográficos de cada país se han tensionado algunos elementos significativos para observar las distancias entre las intervenciones 
Sordini, M. V. (2019). Una lectura sociológica sobre los programas alimentarios en América del Sur. Revista de Sociología, 34(2), 1-19. doi: 10.5354/0719529X.2019.54255

estatales y los datos sobre el problema alimentario. Esta lectura constituye un punto de inicio para indagar el diseño, gestión e implementación de los programas alimentarios de cada país de América del Sur en particular.
Además, será pertinente realizar esos estudios con una mirada longitudinal en tanto desde finales del siglo $\mathrm{XX}$ se ha incrementado la prevalencia de obesidad en la pobreza.

\section{REFERENCIAS}

Adelantado, J., Noguera, J. \& Rambla, X. (2000). El marco de análisis: Las relaciones complejas entre estructura social y políticas sociales. En J. Adelantado (Comp.), Cambios en el estado de bienestar. Políticas sociales y desigualdades en España (pp. 23-62). Barcelona, España: Editorial Icaria.

Aguirre, P. (1990). [El PAN "Programa alimentario nacional" Informe sobre su implementación entre los años 1984-1990]. Documento Técnico Inédito.

Aguirre, P. (2005). Estrategias de consumo: Qué comen los argentinos que comen. Buenos Aires, Argentina: Mino y Dávila.

Aguirre, P. (2011). Reflexiones sobre las nuevas formas del hambre en el siglo XXI: La obesidad de la escasez. Boletín Científico Sapiens Research, 1(2), 60-64.

Aguirre, P. (2015). La situación mundial. En P. Aguirre, D. Díaz Córdova, \& G. Polischer (Eds.), Cocinar y Comer en Argentina Hoy (pp. 23-32). Buenos Aires, Argentina: Sociedad Argentina de Pediatría.

Boito, M. E., \& Huergo, J. (2011). El hambre como punto de origen y de llegada de las políticas alimentarias vigentes. Boletín Científico Sapiens Research, 1(2), 49-53.

Cecchini, S., \& Atuesta, B. (2017). Programas de transferencias condicionadas en América Latina y el Caribe: Tendencias de cobertura $e$ inversión. Serie políticas sociales. Santiago, Chile: CEPAL.

Cena, R. (2014). Programas de transferencias condicionadas de ingresos y programas de empleo en Argentina: Entre la responsabilización de los destinatarios y la individualización de la cuestión social. Boletín Científico Sapiens Research, 4(1), 38.

Danani, C. (2009). La gestión de la política social: Un intento de aportar a su problematización. En M. Chiara, \& M. Di Virgilio (Org.), Gestión de la política social. Conceptos y herramientas (pp. 25-51). Buenos Aires, Argentina: Ed. Prometeo.

De Castro, J. (1955). Geopolítica del hambre. Buenos Aires, Argentina: Editorial Raigal.

De Sena, A. (2011). Promoción de microemprendimientos y políticas sociales: ¿Universalidad, focalización o masividad?, una discusión no acabada. Revista Pensamiento Plural, 8(1), 5-36. doi: 10.15210/pp.v0i8.68

Faleiros V. P. (2000). Las Funciones de la política social en el capitalismo. En E. Borgianni \& C. Montaño (Orgs.), La Política Social Hoy (pp. 43-70). Sao Paulo, Brasil: Cortez Editora.

Organización de las Naciones Unidas para la Alimentación y la Agricultura. (2017). FAOSTAT. Recuperado de: http://www.fao.org/faostat/es/\#country

Garretón, M. (1999). Igualdad, ciudadanía y actores en las políticas sociales. Revista de Ciencias Sociales, $9(1), 41-52$.

Grassi, E. (2003). Políticas y problemas sociales en la sociedad neoliberal. La otra década 
Sordini, M. V. (2019). Una lectura sociológica sobre los programas alimentarios en América del Sur. Revista de Sociología, 34(2), 1-19. doi: 10.5354/0719529X.2019.54255

infame. Buenos Aires, Argentina: Espacio Editorial.

Grassi, E., Hintze, S., \& Neufeld, M. (1994). Políticas sociales, crisis y ajuste. Buenos Aires, Argentina: Espacio Editorial.

Hine, C. (2004). Etnografía virtual. Barcelona, España: Editorial UOC.

Monteiro, C. A., Moura, E. C., Conde, W. L., \& Popkin, B. M. (2004). Socioeconomic status and obesity in adult populations of developing countries: A review. Bulletin of the World Health Organization, 82(12), 940946. doi: S0042-96862004001200011

Organización de las Naciones Unidas para la Alimentación y la Agricultura (2014). Panorama de la seguridad alimentaria y nutricional en América Latina y el Caribe. Hambre en América Latina y el Caribe: Acercándose a los objetivos del milenio. Santiago, Chile: FAO y OPS.

Organización de las Naciones Unidas para la Alimentación y la Agricultura, Fondo Internacional de Desarrollo Agrícola, Organización Mundial de la Salud, Programa Mundial de Alimentos, \& Fondo de las Naciones Unidas para la Infancia (2017). El estado de la seguridad alimentaria y la nutrición en el mundo 2017. Fomentando la resiliencia en aras de la paz y la seguridad alimentaria. Roma, Italia: FAO.

Organización de las Naciones Unidas para la Alimentación y la Agricultura, \& Organización Panamericana de la Salud (2017). Panorama de la seguridad alimentaria y nutricional en América Latina y el Caribe. Santiago, Chile: FAO

Organización Mundial De La Salud (2011). Informe sobre la situación mundial de las enfermedades no transmisibles 2010. Resumen de orientación. Ginebra, Suiza: OMS.

Organización Mundial de la Salud y Organización de las Naciones Unidas para la Alimentación y la Agricultura (2003). Dieta, nutrición y prevención de enfermedades crónicas. Ginebra, Suiza: OMS.

Peña, M., \& Bacallao, J. (2000). La obesidad en la pobreza: Un nuevo reto para la salud pública. Washington, D.C.: OPS.

Plataforma de Seguridad Alimentaria y Nutricional (2019a) Plataforma de seguridad alimentaria. Recuperado de: https://plataformacelac.org/es

Plataforma de Seguridad Alimentaria y Nutricional (2019b). Programa de Alimentación Escolar. Recuperado de: https://plataformacelac.org/programa/296

Plataforma de Seguridad Alimentaria y Nutricional (2019c). Programa Multisectorial Desnutrición Cero. Recuperado de: https://plataformacelac.org/programa/174

Plataforma de Seguridad Alimentaria y Nutricional (2019d). Programa de Atención al Riesgo Nutricional. Recuperado de: https://plataformacelac.org/programa/1243

Plataforma de Seguridad Alimentaria y Nutricional (2019d). Programa Multisectorial de Alimentación y Nutrición en el Ciclo de la Vida. Recuperado de: https://plataformacelac.org/programa/869

Scribano, A., \& De Sena, A. (2013). Los planes de asistencia social en Buenos Aires: Una mirada desde las políticas de los cuerpos y las emociones. Aposta Revista de Ciencias Sociales, 59(2), 1-25.

Sojo, A. (1990). Naturaleza y selectividad de la política social. Revista CEPAL, 41(1), 183199. doi: 10.18356/db9a9bfa-es

Sordini, M. V. (2017). Los programas alimentarios en Argentina desde la sociología del cuerpo/emociones. En A. Scribano \& M. Aranguren (Comp.), Aportes a una sociología de los cuerpos y las emociones desde el Sur (pp. 157-175). Buenos Aires, Argentina: Estudios Sociológicos Editora.

Sordini, M. V. (2018) Las transferencias monetarias de ingresos y el consumo de 
Sordini, M. V. (2019). Una lectura sociológica sobre los programas alimentarios en América del Sur. Revista de Sociología, 34(2), 1-19. doi: 10.5354/0719529X.2019.54255

alimentos en ciudad de Buenos Aires, Argentina. En A. De Sena (ed.), La intervención social en el inicio del siglo XXI: transferencias condicionadas en el orden global (pp. 209-230). Buenos Aires: Estudios Sociológicos Editora.
Titmuss, R. (1981). Política social. Barcelona, España: Editorial Ariel.

Manuscrito recibido: 30-08-2018

Manuscrito aceptado: 22-04-2019 


\section{ANEXO \\ Programas considerados en el estudio}

Se registraron seis programas de Chile (i.e., programa de alimentación escolar; programa de innovación en alimentos + saludables; programa apoyo a familias para el autoconsumo; programa de alimentación complementaria del adulto mayor; programa nacional de alimentación complementaria; programa de escuelas saludables), diez intervenciones en Brasil (i.e., programa de aquisição de alimentos; programa de garantia de preços para a agricultura familiar; programa nacional de fortalecimento da agricultura familiar; programa nacional de suplementação de vitamina a; programa nacional de suplementação de ferro; programa bolsa família; programa de alimentação do trabalhador; programa nacional de alimentação escolar; rede de equipamentos públicos de alimentação e nutrição (restaurantes populares, bancos de alimentos e cozinhas comunitárias; programa nacional de fortalecimento da agricultura familiar), ocho programas de Argentina (i.e., programa de desarrollo rural y agricultura familiar; iniciativa menos sal, más vida; programa generación, sostenimiento $\mathrm{y}$ mejora en la calidad del empleo de agricultores familiares; asignación universal por hijo; plan nacional de seguridad alimentaria, que incluye, entre otros programas, el programa pro huerta y educación alimentaria nutricional; proyecto familias y nutrición; programa abordaje comunitario), seis programas de Bolivia (i.e., programa de alimentación complementaria escolar; programa multisectorial desnutrición cero; programa apoyo a la agricultura familiar comunitaria sustentable; programa multisectorial de alimentación y nutrición en el ciclo de la vida; programa uso y aprovechamiento de la biodiversidad para fines nutricionales; programa educación alimentaria nutricional), tres intervenciones en Colombia (i.e., red de protección social contra la pobreza extrema; programa de alimentación escolar; desayunos infantiles con amor), dos programas de Perú (i.e., programa nacional de alimentación escolar qali warma; programa nacional de apoyo directo a los más pobres), ocho programas alimentarios de Ecuador (i.e., programa provisión de alimentos; proyecto alimentario nutricional integral; programa de atención integral al adulto mayor; programa de alimentación escolar; programa de centros infantiles del buen vivir; programa creciendo con nuestros hijos; proyecto de promoción de alimentos saludables; programa de fortificación casera para la disminución de anemias nutricionales y malnutrición en niños menores de 5 años), ocho intervenciones en Uruguay (i.e., tarjeta Uruguay social; programa de alimentación escolar; asignaciones familiares - plan de equidad; Uruguay crece contigo; sistema nacional de comedores; programa de apoyo a enfermos crónicos; programa de atención al riesgo nutricional; programa nacional de nutrición), nueve programas en Venezuela (i.e., gran misión agro Venezuela; misión 
alimentación; programa de alimentación escolar; misión madres del barrio; programa nutrición para la vida; programa de agricultura familiar y escolar; estrategia espacio laboral activo y nutritivo; gran misión abastecimiento soberano; programa nutripunto) $y$, finalmente, seis programas de Paraguay (i.e., programa de fomento de la producción de alimentos por la agricultura familiar; programa alimentario nutricional integral; pensión alimentaria; proyecto de apoyo a comedores de organizaciones comunitarias; proyecto de inclusión de la agricultura familiar en cadenas de valor; programas de alimentación escolar).

Sistematizar esta información permite realizar una lectura sociológica que tensione los objetivos propuestos por las políticas alimentarias vigentes, los modos de materializar sus intervenciones y la cuestión alimentaria de la región en la actualidad. 